\title{
CRESCIMENTO TUMORAL VERSUS ESTADIAMENTO NO CARCINOMA EPIDERMÓIDE DE LÍNGUA E SOALHO DA BOCA
}

\author{
TUMORAL GROWTH VERSUS STAGING IN SQUAMOUS CELL CARCINOMA \\ OF THE TONGUE AND FLOOR OF THE MOUTH
}

\author{
Ali Amar ${ }^{1}$ \\ Marcos Ribeiro de Magalhães ${ }^{1}$ \\ Lincoln Miyahira ${ }^{2}$ \\ Abrão Rapoport,TCBC/SP ${ }^{3}$ \\ Marcos Brasilino de Carvalho ${ }^{4}$
}

\begin{abstract}
RESUMO: Objetivo: Este estudo tem por objetivo avaliar a relação entre o tamanho do tumor, duração da sintomatologia e a evolução de pacientes com carcinoma epidermóide de língua e soalho bucal. Método: foram avaliados retrospectivamente 226 pacientes submetidos à ressecção de tumor primário de língua e/ou soalho da boca em combinação com esvaziamento cervical. Foi estimado o crescimento mensal do diâmetro tumoral médio (raiz quadrada da superfície/número de meses de evolução) e relacionado com a presença de metástases e recidiva local. A sobrevida livre de doença foi avaliada em relação ao tamanho do tumor e à duração dos sintomas. Resultados: $16 \%$ dos tumores $\mathrm{T} 1$ e T2 e $11 \%$ dos tumores T3 e T4 apresentaram tempo de sintomatologia igual ou inferior a um mês. A mediana de crescimento do diâmetro tumoral foi de $0,81 \mathrm{~cm} / \mathrm{mês}$. Não houve diferença no crescimento tumoral entre os pacientes $\mathrm{pN}+$ ou $\mathrm{pN} 0 ; 30$ pacientes desenvolveram recidiva local, sendo $15 \mathrm{com}$ crescimento tumoral menor do que $0,82 \mathrm{~cm} / \mathrm{mês}$ e 15 no grupo com crescimento $\geq 0,82 \mathrm{~cm} / \mathrm{mês}$. Conclusões: A taxa de crescimento tumoral não se relacionou com o intervalo livre de doença nos pacientes que apresentaram recidiva local. Os pacientes com tumores menos extensos e menor duração dos sintomas apresentaram maior sobrevida livre de doença. O tempo de sintomatologia e a estimativa de crescimento tumoral não apresentam valor prognóstico nos carcinomas epidermóides de língua e soalho oral.
\end{abstract}

Descritores: Crescimento tumoral; Estadiamento; Câncer.

\section{INTRODUÇÃO}

Os pacientes com carcinoma epidermóide da cavidade oral geralmente se apresentam com doença em estádio avançado. Os sinais e sintomas surgem precocemente e a topografia facilita o exame, porém mesmo nos países desenvolvidos, com maior facilidade de acesso ao sistema de saúde, o diagnóstico continua ocorrendo em fases avançadas da doença.

Embora seja esperado um crescimento progressivo do tumor, a falta de relação entre o estadiamento clínico e a duração dos sintomas foi constatada em alguns estu$\operatorname{dos}^{1,2}$. A duração dos sintomas pode ser uma medida imprecisa, no entanto alguns pacientes apresentam tumores extensos com curta sintomatologia enquanto em outros se observa o inverso, e o significado clínico destas diferenças ainda não foi elucidado. A valorização dos sintomas pelo paciente poderia favorecer o diagnóstico precoce, apesar dos sintomas isoladamente serem pouco úteis no diagnóstico do câncer inicial da cavidade oral ${ }^{3,4}$. Este estudo tem por objetivo avaliar o crescimento do tumor, com base na duração dos sintomas, e sua relação com o estádio clínico e o prognóstico.

1. Cirurgião do Serviço de Cirurgia de Cabeça e Pescoço do Hospital Heliópolis, São Paulo, Brasil

2. Residente do Serviço de Cirurgia de Cabeça e Pescoço do Hospital Heliópolis, São Paulo, Brasil

3. Coordenador do Curso de Pós-Graduação do Hospital Heliópolis, São Paulo, Brasil

4. Chefe do Serviço de Cirurgia de Cabeça e Pescoço Hospital Heliópolis, São Paulo, Brasil

Recebido em 09/05/2001

Aceito para publicação em 19/10/2001

Trabalho realizado no Serviço de Cirurgia de Cabeça e Pescoço e Serviço de Cirurgia Geral do Hospital Heliópolis - São Paulo. 


\section{MÉTODO}

Foram avaliados retrospectivamente 226 pacientes com carcinoma epidermóide de língua e/ou soalho oral, tratados no Serviço de Cirurgia de Cabeça e Pescoço do Hospital Heliópolis entre 1978 e 1996. Todos os pacientes receberam tratamento cirúrgico, com ressecção da lesão primária e esvaziamento cervical. A radioterapia adjuvante foi empregada em casos selecionados. Foram excluídos 78 pacientes cujos prontuários estavam incompletos, restando 148 pacientes, dos quais 132 pertenciam ao sexo masculino e 16 ao feminino. A idade média foi de 54 anos (26-80). Quanto ao estadiamento, cinco eram $\mathrm{T}_{1}, 50 \mathrm{~T}_{2}$, $52 \mathrm{~T}_{3}$ e $41\left(\mathrm{~T}_{4}\right)$.

$\mathrm{O}$ crescimento tumoral considerou o diâmetro médio, obtido pela raiz quadrada da superfície tumoral, dividido pelo tempo de duração dos sintomas (Figura 1).

A superfície tumoral foi definida pelo produto dos dois maiores diâmetros perpendiculares do tumor, conforme a descrição contida nos prontuários. A duração dos sintomas considerou o relato do paciente por ocasião da primeira consulta. Para simplificação do cálculo, a dimensão inicial do tumor sintomático foi considerada zero. Os

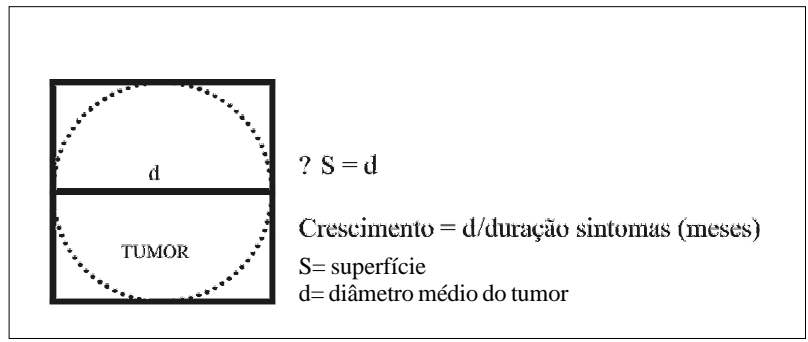

Figura 1. Estimativa de crescimento tumoral. pacientes foram reestadiados conforme os critérios TNM da UICC/AJCC-19975.

O valor mediano do crescimento tumoral ( $\mathrm{cm} / \mathrm{mês})$ foi empregado para definir grupos de crescimento lento ou de crescimento rápido.

Foi analisada a relação entre o crescimento do tumor, o estádio clínico e a metastatização linfática. Entre os pacientes que apresentaram recidiva local, foi avaliado o intervalo livre de doença. A sobrevida livre de doença foi avaliada pelo método atuarial de Kaplan-Meier. A análise estatística empregou testes não-paramétricos: MannWhitney, Kruskal-Wallis e log-rank.

\section{RESULTADOS}

Quanto ao tempo de sintomatologia, 16\% dos tumores de estádio T1 e T2 e 11\% dos tumores de estádio T3 e T4 apresentaram duração dos sintomas igual ou inferior a um mês por ocasião da primeira consulta com o especialista. A Figura 2 mostra a duração dos sintomas em relação aos diferentes estadiamentos do tumor primário.

A duração dos sintomas apresentou mediana de três meses (1-24) e a superfície tumoral apresentou mediana de $12 \mathrm{~cm}^{2}$ (1-80). A mediana do crescimento do diâmetro tumoral foi de $0,81 \mathrm{~cm} / \mathrm{mês}\left(\mathrm{Q}_{25-75 \%}=0,53-1,73\right)$. $O$ crescimento tumoral mostrou sobreposição de valores entre os diferentes estádios T (Figura 3).

A taxa de crescimento apresentou mediana de $0,72 \mathrm{~cm} / \mathrm{mês}\left(\mathrm{Q}_{25-75 \%}=0,53-1,54\right)$ nos tumores metastáticos $(\mathrm{pN}+)$ e $0,99 \mathrm{~cm} / \mathrm{mês}\left(\mathrm{Q}_{25-75 \%}=0,50-1,93\right)$ nos tumores não-metastáticos (pN0).

Quanto à evolução, 30 pacientes apresentaram recidiva local, sendo que 15 cursaram com tumores com crescimento inferior a $0,82 \mathrm{~cm} / \mathrm{mês}$ e 15 com tumores de crescimento igual ou superior a $0,82 \mathrm{~cm} / \mathrm{mês}$. As recidivas

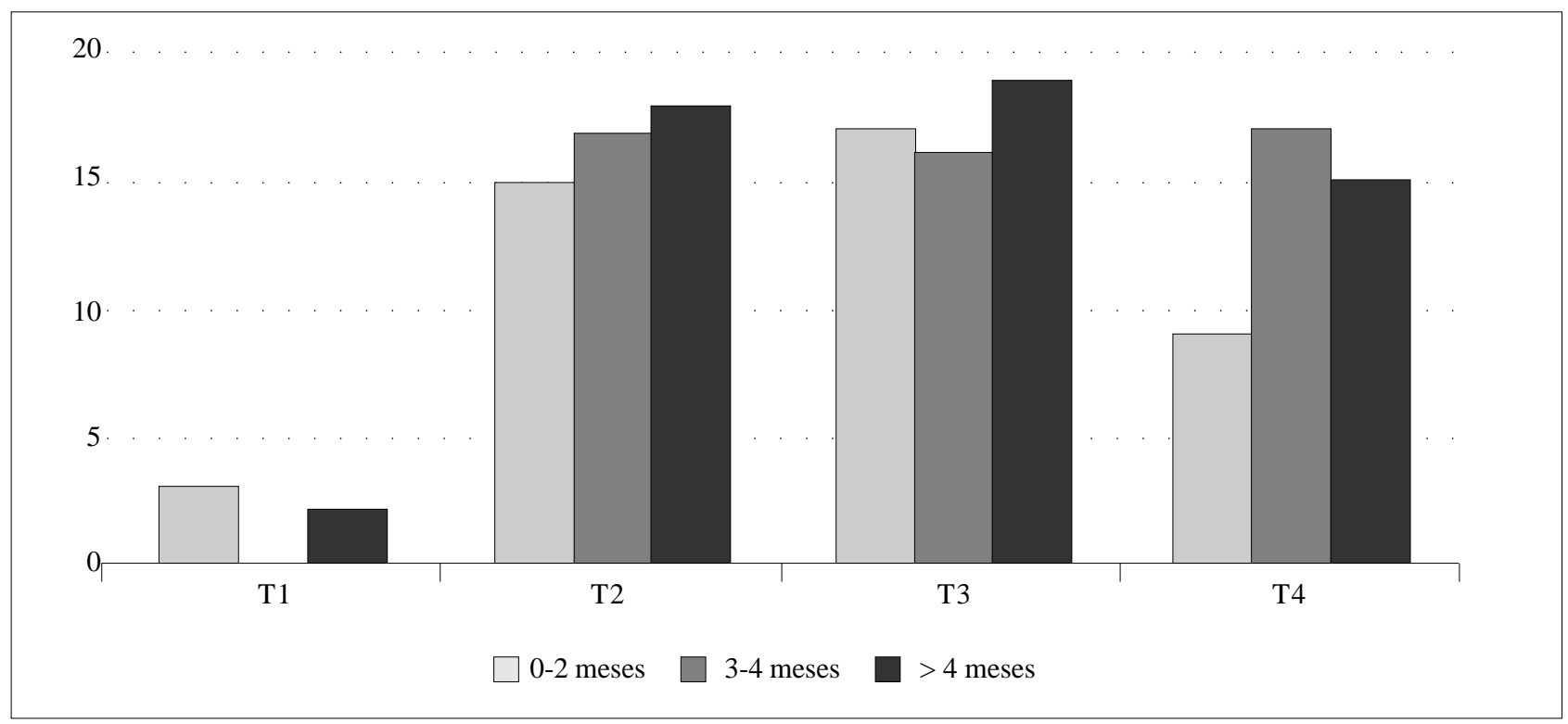

Figura 2. Estadiamento e duração dos sintomas. 
ocorreram mais precocemente nos pacientes que apresentaram tumores com menor taxa de crescimento, porém sem significância estatística (Figura 4).

Não houve diferença na sobrevida livre de doença entre os pacientes com estimativa de crescimento inferior a $0,82 \mathrm{~cm} / \mathrm{mês}$ e aqueles com crescimento igual ou superior a $0,82 \mathrm{~cm} / \mathrm{mês}$; a sobrevida a cinco anos nestes dois grupos foi de $51 \%$ e $49 \%$ respectivamente $(\mathrm{p}=0,81)$. Os tumores com menor área e duração dos sintomas apresentaram maior sobrevida livre de doença (Figura 5).

\section{DISCUSSÃO}

A duração dos sintomas é uma estimativa imprecisa da evolução do tumor, além de desconsiderar um importante período de evolução subclínica, a informação do paciente pode ser inexata ou mal avaliada pelo médico.

O maior crescimento do tumor pode ter várias causas, como maior proliferação ou menor índice de morte celular, a coalescência de focos multicêntricos originada na cancerização de campo, ou ainda as características de

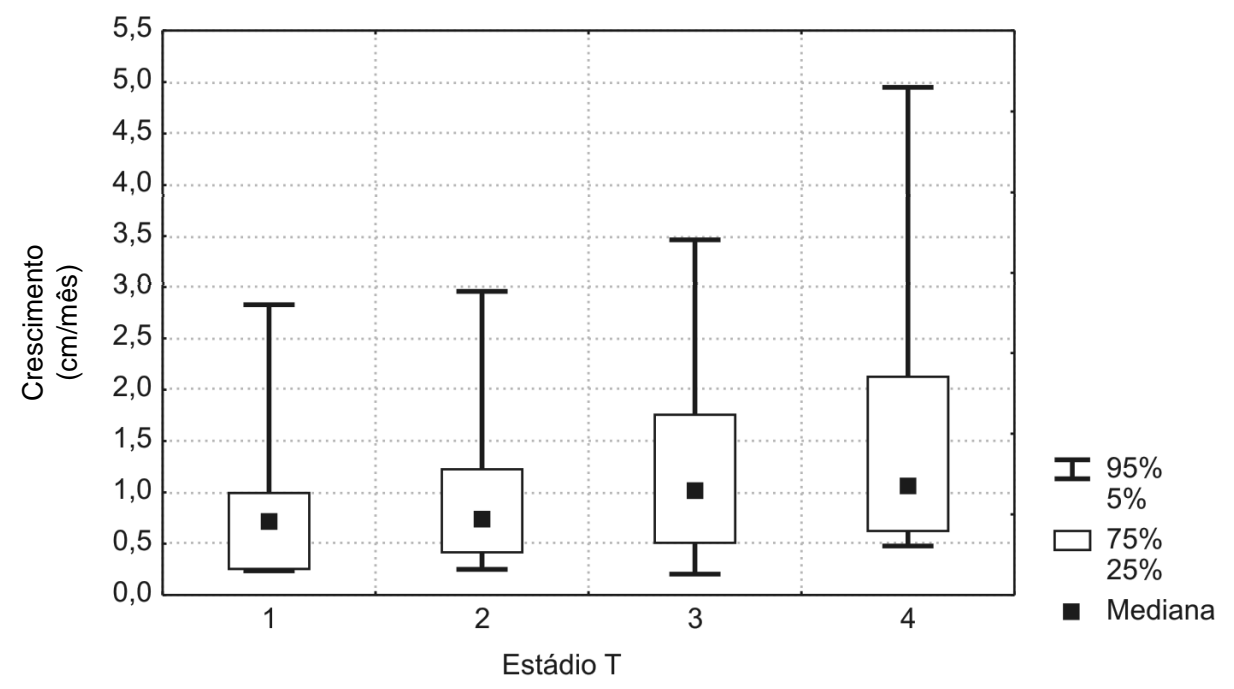

Figura 3. Crescimento tumoral.

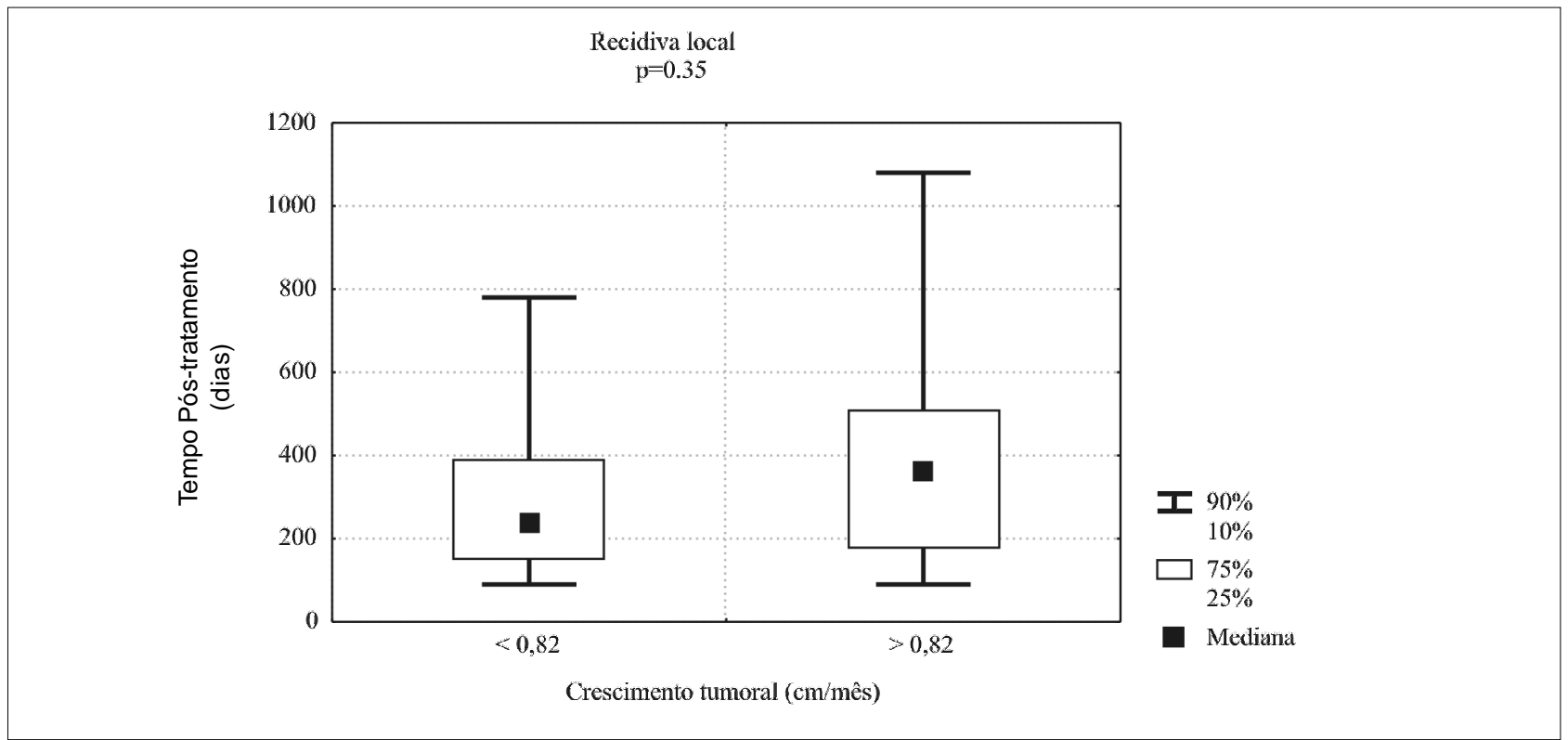

Figura 4. Crescimento tumoral e recidiva local. 


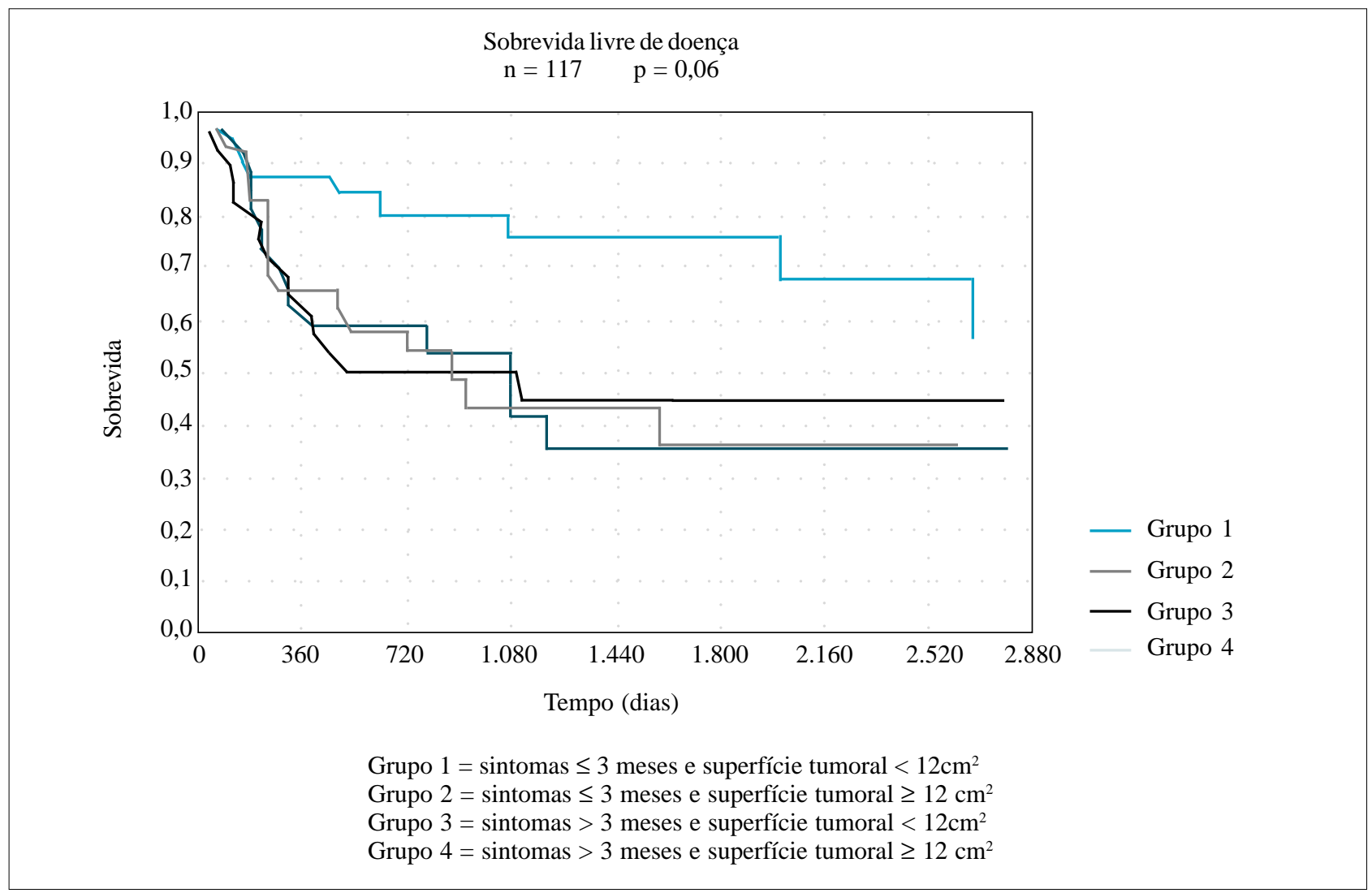

Figura 5. Sobrevida em relação a duração dos sintomas e superfície tumoral.

interação do tumor com o tecido adjacente (células normais e estroma), que determina a sua capacidade de invasão $0^{6,7}$.

A estimativa de crescimento tumoral não teve valor prognóstico. O maior tamanho ou o maior tempo de permanência do tumor são fatores que podem se relacionar com pior prognóstico, mas a fórmula empregada fez com que estas variáveis se anulassem (tamanho/tempo). Lesões extensas de longa duração apresentam taxas de crescimento baixas, assim como tumores pequenos de curta duração podem apresentar taxas de crescimento elevadas. Os tumores também apresentam redução em seu crescimento logarítmico após atingirem volumes maiores. Houve grande sobreposição entre as estimativas de crescimento para os diferentes estádios T (Figura 1). Conforme pode ser observado na Figura 5, os tumores extensos apresentam evolução desfavorável independentemente da duração dos sintomas. Entre os tumores menores $\left(<12 \mathrm{~cm}^{2}\right)$, aqueles com menor duração dos sintomas apresentaram sobrevida livre de doença maior. A falta de relação entre o estadiamento e o tempo de evolução clínica sugere que o tamanho do tumor reflita um estado de equilíbrio momentâneo. Assim, uma abordagem terapêutica precoce poderia simplificar o tratamento, porém sem a garantia de um impacto significativo sobre os resultados. Outros estudos também não encontraram associação entre a duração dos sintomas e o estadiamento do tumor, embora um atraso superior a um mês acarrete maior probabilidade de diagnóstico em estádio mais avançado ${ }^{8}$. Diante desta perspectiva, é questionável a realização de rastreamento da população de risco, uma estratégia que exige acompanhamento mensal, pois o câncer de boca ainda apresenta baixa incidência.

A estimativa de crescimento tumoral também não permitiu identificar os pacientes com metástases ou aqueles com recidivas precoces. Isto poderia ocorrer tanto pela diferença de comportamento entre tumores micro e macroscópicos, como por características biológicas distintas entre o tumor primário ressecado e o tumor residual.

A velocidade de crescimento do tumor, com mediana de $0,81 \mathrm{~cm} / \mathrm{mês}$ (diâmetro médio), não pode ser considerada uma progressão rápida, o que contrasta com o predomínio de lesões em estádio avançado.

Embora seja razoável considerar uma evolução progressiva do estádio T1 para T2 e T3, sendo que qualquer um destes estádios pode evoluir para $\mathrm{T} 4$, o estadiamento TNM expressa melhor as características biológicas do tumor do que a sua evolução temporal. 


\begin{abstract}
Background: The objective of this study is to evaluate the real impact of tumor size, on symptoms and evolution of patients with squamous cell carcinoma of the tongue and floor of the mouth. Methods: after 226 neck dissections plus primary resection, were estimated the monthly growing of the medium tumor diameter (square root of surface/number of months) and related with metastasis presence and local recurrence. The free disease survival were analysed in relation to tumor syze and complaints length.. Results: $16 \%$ of T1 and T2 and 11\% of T3 and $T 4$ tumors growth of tumoral diameter were $0,81 \mathrm{~cm} / \mathrm{month}$, without differences between $\mathrm{pN}+$ and pNO patients; 30 patients with local recurrence, being 15 with tumoral growing less than $0,82 \mathrm{~cm} / \mathrm{month}$ and 15 with more than $0,82 \mathrm{~cm} /$ month. Conclusions: The neoplasia growing were not related with free disease survival in patients with local recurrence, and those with less extensive tumors and complaints time present a greater free disease survival. The time complaints and the tumoral growing were not prognostic in squamous cell carcinoma of tongue and flon of the mouth.
\end{abstract}

Key words: Tumoral growing; Staging; Cancer.

\section{REFERÊNCIAS}

1. Dhooge Ij, Albers Fw, Van Cauwenberge Pb. Clinical characteristics and diagnostic delay of head and neck cancer; results from a prospective study in Belgium. Eur J Surg Oncol 1996; 354-35.

2. Vernham GA, Crowther JA. Head and neck carcinoma: stage at presentation. Clin Otolaryngol 1998, 19:120-4.

3. Dolan RW, Vaughan CW, Fuleihan N. Symptoms in early head and neck cancer: an inadequate indicator. Otolaryngol Head Neck Surg 1998, 119:463-7.

4. Allison P, Franco E, Feine J. Predictors of professional diagnostic delays for upper aerodigestive tract carcinoma. Oral Oncol 1998, 34:127-32.

5. Sobin LH, Wittekind C. TNM-Classification of Malignant Tumors. 5 ed. Wiley-Liss, 1997.

6. Slaughter DP, Southwick HW, Smejkal W. Field cancerization in oral stratified squamous epithelium. Cancer 1953, 6:963-8.

7. van Oijen MGCT, Slootweg PJ. Oral field cancerization: carcinogen-induced independent events or micrometastatic deposits? Cancer Epidemiol Biomarkers Prev 2000, 9:249-56.
8. Allison P, Franco E, Black M, Feine J. The role of professional diagnostic delays in the prognosis of upper aerodigestive tract carcinoma. Oral Oncol 1998, 34:147-53.

Endereço para correspondência:

Prof. Dr. Abrão Rapoport

Praça Amadeu Amaral, 47 - cj. 82

01327-010 — São Paulo-SP 